\title{
Composite Exponential Approximations
}

\author{
By Arieh Iserles
}

\begin{abstract}
The Composite Exponential Approximations (CEA) arise in a natural way when one investigates the stability and order properties of a combination of several methods for the numerical solution of ordinary differential equations, sequentially implemented with different step-lengths. Some general results on the order, acceptability and exponential fitting properties of CEA are derived. The composite Padé approximations and $\boldsymbol{N}$-approximations are explored in detail.
\end{abstract}

1. Introduction. This paper gives a theory which is relevant to the use of variable step-length to increase the order of solution of stiff ordinary differential systems. In a nutshell, we explore the effect of combining two (or more) different numerical methods with different step-lengths so that the numerical order is increased, while other desirable properties of the solution (stability, exponential fitting, etc.) are retained.

In its spirit this work follows two trails: first, the cyclic linear multistep methods [2], [20]. These methods consist of sequential application of several (possibly zero-unstable) linear multistep schemes, each with a constant step-length, so that the outcome, as a whole, is zero-stable and of high order. Second, the use of an a priori determined sequence of step-lengths (with a single method) in order to try and minimize the global error [6], [7], [13], [15].

In the sequel, instead of considering the methods themselves, we examine exponential approximations, where an exponential approximation (stability function) which corresponds to a given method is the solution by the method of the linear scalar test equation $y^{\prime}=a y, y(0)=1$, with unit step-length. The theory of these composite exponential approximations is much more general and uniform than separate examination of each family of numerical methods, and it answers directly the questions of $A$-stability and exponential fitting. As far as order is concerned, the way back from exponential approximations to methods is less straightforward. It will be described in detail in a forthcoming paper, which gives particular attention to Obrechkoff, Adams-Nørsett, implicit and semiexplicit Runge-Kutta methods. By using the given theory of composite exponential approximations that paper will develop numerical methods which have order, $A$ stability and exponential fitting properties superior to the existing schemes. For example, it will be shown that a two-stage $A$-stable semiexplicit Runge-Kutta process of order four and an exponentially fitted $\nu$-stage $A$-stable implicit RungeKutta scheme of order $2 \nu$ can be obtained.

Received November 25, 1980.

1980 Mathematics Subject Classification. Primary 65L05, 41 A20. 
In Section 2 we examine the basic model of composite exponential approximations, give some existence results and explore the connection between them and the concept of dominant pairs [8].

Section 3 is devoted to Padé approximations and to their generalizations, while Section 4 investigates the Nørsett approximations.

2. Composite Exponential Approximations. Let $R_{1}, \ldots, R_{N}$ be rational approximations to $\exp (x)$ such that $R_{i}(x)-\exp (x)=O\left(|x|^{p+1}\right), i=1, \ldots, N$, and let $d_{1} \ldots, d_{N}$ be arbitrary positive numbers such that $\sum_{i=1}^{N} d_{i}=1$. Then the Composite Exponential Approximation (CEA) is defined as

$$
R\left(x ; d_{1}, \ldots, d_{N}\right)=R_{1}\left(d_{1} x\right) R_{2}\left(d_{2} x\right) \cdots R_{N}\left(d_{N} x\right) .
$$

It follows that $R\left(x ; d_{1}, \ldots, d_{N}\right)-\exp (x)=O\left(|x|^{p+1}\right)$.

We restrict our attention to positive $d_{i}$ 's, because they correspond to forward integration of a system of ordinary differential equations.

In the sequel we will be interested in the following problems:

(i) order: whether $d_{1}, \ldots, d_{N}$ exist so that $R\left(x ; d_{1}, \ldots, d_{N}\right)-\exp (x)=$ $O\left(|x|^{p+s+1}\right)$ for $s \geqslant 1$, in particular for $s=N$.

(ii) exponential fitting: whether $d_{1}, \ldots, d_{N}$ exist so that the equation $R\left(\lambda ; d_{1}, \ldots, d_{N}\right)=\exp (\lambda)$ holds for certain negative real values of $\lambda$.

(iii) $A$-acceptability: whether the approximation $R\left(x ; d_{1}, \ldots, d_{N}\right)$ is $A$ acceptable, where we remind the reader that the exponential approximation $R$ is $A$ acceptable if $|R(z)| \leqslant 1$ for every complex number $z$ such that $\operatorname{Re} z \leqslant 0$.

In some cases, when it makes sense from the numerical point of view, we consider the less stringent $A_{0}$-acceptability criterion, which is that $|R(x)| \leqslant 1$ for all nonpositive real values of $x$.

The following sufficient condition for $A$-acceptability is elementary.

LemMA 1. If $R_{i}, i=1, \ldots, N$, are A-acceptable $\left(A_{0^{-}}\right.$-acceptable $)$, then $R$ is A-acceptable ( $A_{0}$-acceptable).

The connection between the $d_{k}$ 's and the order of the CEA is central to the given theory. Let

$$
R_{k}(x)-\exp (x)=\sum_{q=p+1}^{\infty} \alpha_{k, q} x^{q}, \quad 1 \leqslant k \leqslant N
$$

and

$$
R\left(x ; d_{1}, \ldots, d_{N}\right)-\exp (x)=\sum_{q=p+1}^{\infty} A_{q}\left(d_{1}, \ldots, d_{N}\right) x^{q}
$$

THEOREM 2. Let $R_{1}, \ldots, R_{N}$ be rational exponential approximations of order $p$ and let $s$ be an integer between 1 and $p+1$. Then the $C E A R\left(x ; d_{1}, \ldots, d_{N}\right)=$ $\Pi_{k=1}^{N} R_{k}\left(d_{k} x\right)$ is of order $p+s$ at least if and only if

$$
\sum_{k=1}^{N}\left(\sum_{i=0}^{r-p-1} \frac{(-1)^{i}}{i !} \alpha_{k, r-i}\right) d_{k}^{r}=0, \quad p+1<r \leqslant p+s .
$$


Proof. We set

$\tilde{d}_{k}=d_{k} / \sum_{j=1}^{N-1} d_{j}, \quad 1 \leqslant k \leqslant N-1$, and $\tilde{R}\left(x ; \tilde{d}_{1}, \ldots, \tilde{d}_{N-1}\right)=\prod_{k=1}^{N-1} R_{k}\left(\tilde{d}_{k} x\right)$

and assume that

$$
\tilde{R}\left(x ; \tilde{d}_{1}, \ldots, \tilde{d}_{N-1}\right)=\sum_{q=p+1}^{\infty} \tilde{A}_{q}\left(\tilde{d}_{1}, \ldots, \tilde{d}_{N-1}\right) x^{q} .
$$

We are interested in deriving an explicit form of $A_{q}$ as a function of the $\alpha_{k, q}$ 's and the $d_{k}$ 's. First we find a relation between $A_{q}$ and $\tilde{A}_{q}$ :

$$
\begin{aligned}
& \sum_{q=p+1}^{\infty} A_{q}\left(d_{1}, \ldots, d_{N}\right) x^{q} \\
&=\prod_{k=1}^{N} R_{k}\left(d_{k} x\right)-e^{x}=\prod_{k=1}^{N-1} R_{k}\left(\tilde{d}_{k} \sum_{j=1}^{N-1} d_{j} x\right) R_{N}\left(d_{N} x\right)-e^{x} \\
&=\left\{\exp \left(\sum_{j=1}^{N-1} d_{j} x\right)+\sum_{q=p+1}^{\infty} \tilde{A}_{q}\left(\tilde{d}_{1}, \ldots, \tilde{d}_{N-1}\right)\left(\sum_{j=1}^{N-1} d_{j}\right)^{q} x^{q}\right\} \\
& \times\left\{e^{d_{N} x}+\sum_{q=p+1}^{\infty} \alpha_{N, q} d_{N}^{q} x^{q}\right\}-e^{x} .
\end{aligned}
$$

Therefore, for every $p+1 \leqslant q \leqslant 2 p+1, \sum_{j=1}^{N} d_{j}=1$ implies

$$
\begin{aligned}
A_{q}\left(d_{1}, \ldots, d_{N}\right)= & \sum_{i=0}^{q-p-1} \frac{1}{i !} \tilde{A}_{q-i}\left(\tilde{d}_{1}, \ldots, \tilde{d}_{N-1}\right)\left(\sum_{j=1}^{N-1} d_{j}\right)^{q-i} d_{N}^{i} \\
& +\sum_{i=0}^{q-p-1} \frac{1}{i !} \alpha_{N, q-i} d_{N}^{q-i}\left(\sum_{j=1}^{N-1} d_{j}\right)^{i} \\
= & \sum_{i=1}^{q-p-1} \frac{1}{i !}\left\{d_{N}^{q-i}\left(1-d_{N}\right)^{i} \alpha_{N, q-i}\right. \\
& \left.\quad+\left(1-d_{N}\right)^{q-i} d_{N}^{i} \tilde{A}_{q-i}\left(\tilde{d}_{1}, \ldots, \tilde{d}_{N-1}\right)\right\} .
\end{aligned}
$$

But firstly, the order of multiplication in the definition of $R$ does not matter, and so $A_{q}$ is symmetric in $\left(\left\{\alpha_{k, r}\right\}_{r=p+1}^{\infty}, d_{k}\right), 1 \leqslant k \leqslant N$. Secondly, the $\alpha_{k, q}$ 's are independent of the $d_{k}$ 's. Hence

$$
A_{q}\left(d_{1}, \ldots, d_{N}\right)=\sum_{i=0}^{q-p-1} \frac{1}{i !} \sum_{k=1}^{N} \alpha_{k, q-i} d_{k}^{q-i}\left(1-d_{k}\right)^{i}
$$

for every $p+1 \leqslant q \leqslant 2 p+1$.

Let

$$
B_{r}\left(d_{1}, \ldots, d_{N}\right)=\sum_{j=0}^{N} \sum_{i=0}^{r-p-1} \frac{(-1)^{i}}{i !} \alpha_{j, r-1} d_{j} \quad \text { for } p+1<r \leqslant 2 p+1
$$


By changing the order of summation and shift of indices, for every $p+1<q<$ $2 p+1$,

$$
\begin{aligned}
A_{q}\left(d_{1}, \ldots, d_{N}\right) & =\sum_{i=0}^{q-p-1} \frac{1}{i !} \sum_{k=1}^{N} \alpha_{k, q-i} d_{k}^{q-i} \sum_{r=0}^{i}\left(\begin{array}{l}
i \\
r
\end{array}\right)(-1)^{r} d_{k} \\
& =\sum_{r=p+1}^{q}(-1)^{r} \sum_{i=q-r}^{q-p-1} \frac{(-1)^{q-i}}{i !}\left(\begin{array}{c}
i \\
r-q+i
\end{array}\right) \sum_{k=1}^{N} \alpha_{k, q-i} d_{k}^{r} \\
& =\sum_{r=p+1}^{q} \frac{(-1)^{r}}{(q-r) !} \sum_{i=0}^{r-p-1} \frac{(-1)^{i+r}}{i !} \sum_{k=1}^{N} \alpha_{k, r-i} d_{k}^{r} \\
& =\sum_{r=p+1}^{q} \frac{1}{(q-r) !} B_{r}\left(d_{1}, \ldots, d_{N}\right) .
\end{aligned}
$$

Let us suppose that $R\left(x ; d_{1}, \ldots, d_{N}\right)-\exp (x)=O\left(x^{p+s+1}\right)$, where $1<s<$ $p+1$. Then $A_{q}\left(d_{1}, \ldots, d_{N}\right)=0$ for every $q$ between $p+1$ and $p+s$. But

$$
A_{q}\left(d_{1}, \ldots, d_{N}\right)=\sum_{r=p+1}^{q} \frac{1}{(q-r) !} B_{r}\left(d_{1}, \ldots, d_{N}\right), \quad p+1 \leqslant q \leqslant p+s
$$

is a triangular nonsingular linear algebraic system. Hence $B_{r}\left(d_{1}, \ldots, d_{N}\right)=0$, $p+1<r \leqslant p+s$, which is the desired result.

We use the notation $a_{k}=\alpha_{k, p+1}, b_{k}=\alpha_{k, p+2}, 1 \leqslant k \leqslant N$. Hence $R_{k}(x)-\exp (x)$ $=a_{k} x^{p+1}+b_{k} x^{p+2}+O\left(|x|^{p+3}\right)$. By Theorem 2 the necessary and sufficient condition for a CEA of order $p+1$ is

$$
\sum_{k=1}^{N} a_{k} d_{k}^{p+1}=0
$$

while the attainment of order $p+2$ is equivalent to (2.2) together with

$$
\sum_{k=1}^{N}\left(b_{k}-a_{k}\right) d_{k}^{p+2}=0
$$

If $N=2$, the condition (2.2) immediately yields the following result.

THEOREM 3. If $R_{k}(x)-\exp (x)=a_{k} x^{p+1}+O\left(x^{p+2}\right), a_{k} \neq 0, k=1,2$, then

(a) If $a_{1} a_{2}>0$, then no positive $d_{k}$ 's exist so that $R$ is of order $p+1$.

(b) If $a_{1} a_{2}<0$, then there is a unique positive pair $\left(d_{1}, d_{2}\right)$ both in the interval $(0,1)$ and given by

$$
\begin{aligned}
& d_{1}=\left(-a_{2} / a_{1}\right)^{1 /(p+1)} /\left(1+\left(-a_{2} / a_{1}\right)^{1 /(p+1)}\right), \\
& d_{2}=1 /\left(1+\left(-a_{2} / a_{1}\right)^{1 /(p+1)}\right),
\end{aligned}
$$

such that $R$ is of order $p+1$.

Proof. By an examination of (2.2) for $N=2$, namely

$$
a_{1} d_{1}^{p+1}+a_{2} d_{2}^{p+1}=0, \quad d_{1}+d_{2}=1 .
$$

No transparent existence and uniqueness result with the same scope as Theorem 3 has been found for $N=3$, but the following statement is useful. 
THEOREM 4. Let $R_{k}(x)-\exp (x)=a_{k} x^{p+1}+b_{k} x^{p+2}+O\left(|x|^{p+3}\right), a_{k} \neq 0, k=$ $1,2,3$, and let the products $a_{1} a_{2}$ and $a_{2} a_{3}$ be negative. If either

$$
b_{2}+\eta^{p+2} b_{1}>(1-\eta) a_{2}, \quad b_{3}+\sigma^{p+2} b_{2}<(1-\sigma) q_{3},
$$

where $\eta=\left(-a_{2} / a_{1}\right)^{1 /(p+1)}, \sigma=\left(-a_{3} / a_{2}\right)^{1 /(p+1)}$ or these two inequalities are reversed, then $d_{1}, d_{2}, d_{3}$ in $(0,1)$ exist, $d_{1}+d_{2}+d_{3}=1$, such that $R$ is of order $p+2$.

Proof. We set for $\theta$ in $[0,1]$

$$
\begin{aligned}
& d^{*}(\theta)=\left\{-\left(a_{1} \theta^{p+1}+a_{3}(1-\theta)^{p+1}\right) / a_{2}\right\}^{1 /(p+1)}, \\
& d_{1}^{*}(\theta)=\theta /\left(1+d^{*}(\theta)\right) \\
& d_{2}^{*}(\theta)=d^{*}(\theta) /\left(1+d^{*}(\theta)\right) \\
& d_{3}^{*}(\theta)=(1-\theta) /\left(1+d^{*}(\theta)\right)
\end{aligned}
$$

and

$$
R^{*}(x ; \theta)=R_{1}\left(d_{1}^{*}(\theta)\right) R_{2}\left(d_{2}^{*}(\theta)\right) R_{3}\left(d_{3}^{*}(\theta)\right) .
$$

It is easily seen that $\Sigma_{k=1}^{3} a_{k} d_{k}^{* p+1}(\theta)=0$ for every $\theta \in[0,1]$. Therefore, by (2.2), $R^{*}$ is of order $p+1$ for every $\theta$ between 0 and 1 .

Let $\xi(\theta)=A_{p+2}\left(d_{1}^{*}(\theta), d_{2}^{*}(\theta), d_{3}^{*}(\theta)\right)$. By Theorem 2

$$
\begin{aligned}
\xi(\theta) & =B_{p+2}\left(d_{1}^{*}(\theta), d_{2}^{*}(\theta), d_{3}(\theta)\right)=\sum_{k=1}^{3}\left(b_{k}-\xi_{k}\right)\left[d_{k}^{*}(\theta)\right]^{p+2} \\
& =\frac{\left\{\left(b_{1}-a_{1}\right) \theta^{p+2}+\left(b_{2}-a_{2}\right)\left[d^{*}(\theta)\right]^{p+2}+\left(b_{3}-a_{3}\right)(1-\theta)^{p+2}\right\}}{\left(1+d^{*}(\theta)\right)^{p+2}} .
\end{aligned}
$$

But $d^{*}(0)=\sigma, d^{*}(1)=\eta$, and so

$$
\begin{aligned}
& \xi(0)=\left\{b_{2} \sigma^{p+2}+b_{3}-a_{3}(1-\sigma)\right\} /(1+\sigma)^{p+2} \\
& \xi(1)=\left\{b_{1}+b_{2} \eta^{p+2}-a_{1}(1-\eta)\right\} /(1+\eta)^{p+2}
\end{aligned}
$$

Therefore $\xi(0) \xi(1)<0$, because of (2.4) and because $\sigma$ and $\eta$ are positive. Hence, by the continuity of $\xi$, there is $\theta_{0}$ in $(0,1)$ such that $\xi\left(\theta_{0}\right)=0$ and $R^{*}\left(x, \theta_{0}\right)$ is the desired CEA of order $p+2$.

Observe that conditions of the form $a_{i} a_{j}<0$ appear in both Theorems 3 and 4. Many useful pairs of exponential approximations satisfy them.

Following [8] we call $\left\{R_{1}, R_{2}\right\}$ a dominant pair if for every $x \leqslant 0$

$$
\min \left\{R_{1}(x), R_{2}(x)\right\} \leqslant e^{x} \leqslant \max \left\{R_{1}(x), R_{2}(x)\right\} .
$$

If $\left\{R_{1}, R_{2}\right\}$ is dominant, then $a_{1} a_{2}<0$. Hence we can use in the sequel the results of [8] and [10] to determine some exponential approximations that satisfy the conditions of Theorems 3 and 4.

In exponential fitting [14] the concept of dominant pairs is useful because of the following theorem.

TheOREM 5. Let $\left\{R_{1}, R_{2}\right\}$ be a dominant pair. Then for every $x_{0}<0$, a $d=d\left(x_{0}\right)$ in $(0,1)$ exists such that $R\left(x_{0} ; d, 1-d\right)=\exp \left(x_{0}\right)$. 
Proof. (2.5) implies that $\exp \left(x_{0}\right)$ is in the interval whose endpoints are $R\left(x_{0} ; 0,1\right)$ and $R\left(x_{0} ; 1,0\right)$. The required result follows from the fact that $R\left(x_{0} ; d, 1-d\right)$ is a continuous function of $d$.

Two possible algorithms for computing the number $d\left(x_{0}\right)$ of Theorem 5 are:

(a) To approximate $d\left(x_{0}\right)$ by a rational function initially and then to iterate by the Newton-Raphson method.

(b) To use bisection, based on the fact that $d\left(x_{0}\right)$ is in the interval $[0,1]$.

3. Padé-Type Approximations. The Padé approximations to $\exp (x)$ have the form

$$
\begin{aligned}
& R_{n, m}(x)=P_{n, m}(x) / Q_{n, m}(x), \\
& P_{n, m}(x)=\sum_{k=0}^{m} \frac{(n+m-k) ! m !}{(n+m) ! k !(m-k) !} x^{k}, \quad Q_{n, m}(x)=P_{m, n}(-x),
\end{aligned}
$$

and they satisfy $R_{n, m}(x)-\exp (x)=O\left(x^{n+m+1}\right)$. According to [3], [21], and [22] the only $A$-acceptable Padé approximations are $R_{n, n}, R_{n+1, n}, R_{n+2, n}, n=0,1, \ldots$, while all the approximations $R_{n, m}, n \geqslant m$, are $A_{0}$-acceptable. According to [8] the pair $\left\{R_{n_{1}, m_{1}}, R_{n_{2}, m_{2}}\right\}$ is dominant if and only if $m_{1}+m_{2}$ is odd.

LEMMA 6. The coefficients of the equation

$$
R_{n, m}(x)-\exp (x)=a_{n, m} x^{n+m+1}+b_{n, m} x^{n+m+2}+O\left(x^{n+m+3}\right)
$$

have the values

$$
\begin{aligned}
& a_{n, m}=(-1)^{n-1} \frac{m ! n !}{(n+m) !(n+m+1) !} \\
& b_{n, m}=(-1)^{n-1} \frac{m ! n !\left(2 n^{2}+2 n m+3 n+m\right)}{(n+m) !(n+m+2) !(n+m)} .
\end{aligned}
$$

Proof. From [9] we obtain

$$
P_{n, m}(x)-e^{x} Q_{n, m}(x)=(-1)^{n-1} \sum_{k=n+m+1}^{\infty} \frac{m !(k-m-1) !}{(n+m) ! k !(k-n-m-1) !} x^{k} .
$$

The result follows from this equation and from

$$
\left[Q_{n, m}(x)\right]^{-1}=1+\frac{n}{n+m} x+O\left(x^{2}\right) .
$$

Particular cases of CEA composed of two Padé approximations can be treated using Theorem 3 and Lemma 6. For instance the following two pairs of Padé approximations are of interest:

(a) $R_{1}=R_{n, n}, R_{2}=R_{n+1, n-1}$.

In this case $d_{1}=d^{*} /\left(1+d^{*}\right), d_{2}=1 /\left(1+d^{*}\right)$, where $d^{*}=(1+1 / n)^{1 /(2 n+1)}$. The CEA is of order $2 n+1$ and it is $L$-acceptable (i.e., $A$-acceptable and tending to zero as $\operatorname{Re} x \rightarrow-\infty)$.

(b) $R_{1}=R_{n, n-1}, R_{2}=R_{n-1, n}$.

In this case $d_{1}=d_{2}=\frac{1}{2}$. The CEA is of order $2 n$. Observe that $R_{n-1, n}$ cannot be even $A_{0}$-acceptable, but Theorem 9, which is stated and proved later, implies that the CEA is $\boldsymbol{A}$-acceptable. 
(c) Theorem 4 gives a sufficient condition for the existence of a CEA of order $p+2$ composed out of three Padé approximations. If $R_{1}=R_{n-1, m+1}, R_{2}=R_{n, m}$, $R_{3}=R_{n+1, m-1}$, then $a_{1} a_{2}, a_{2} a_{3}<0$ and after some algebra the conditions of the conclusion reduce to $A_{1}(n, m) A_{2}(n, m)>0$, where

$$
\begin{aligned}
& A_{1}(n, m)=2\left(\frac{n}{m+1}\right)^{1 /(n+m+1)}-(n-m)\left(\left(\frac{n}{m+1}\right)^{1 /(n+m+1)}-1\right) \\
& A_{2}(n, m)=2-(n-m)\left(\left(\frac{n+1}{m}\right)^{1 /(n+m+1)}-1\right)
\end{aligned}
$$

It can be shown that $A_{1}(n, m)$ and $A_{2}(n, m)$ are positive for every natural $n$ and $m$. Therefore a CEA of this type exists for every choice of $n$ and $m$.

In particular, according to Lemma $6, a_{n n}=b_{n n}$. Hence, if $R_{n, n}$ appears among the $R_{k}$ 's, it is straightforward to calculate the $d_{k}$ 's that satisfy formulae (2.2) and (2.3). For instance, when $m=n$, we have the following case:

(c') $R_{1}=R_{n-1, n+1}, R_{2}=R_{n, n}, R_{3}=R_{n+1, n-1}$.

The conditions (3.1) are satisfied. Furthermore, from (2.4), (2.5) we derive

$$
d_{1}=d_{3}=d^{*} /\left(1+2 d^{*}\right), \quad d_{2}=1 /\left(1+2 d^{*}\right),
$$

where $\left.d^{*}=(n /(2(n+1)))\right)^{1 /(2 n+1)}$.

Hence $R\left(x ; d_{1}, d_{2}, d_{3}\right)$ is of order $2 n+2$.

LEMma 7. The CEA $R$, as defined above, is A-acceptable.

Proof. Because of the maximum modulus theorem, it is sufficient to prove that $R$ is analytic in the complex left half-plane and that $\left|R\left(i t ; d_{1}, d_{2}, d_{3}\right)\right|^{2} \leqslant 1$ for every real $t$. Because the $d_{k}$ 's are positive the analyticity of the $R_{k}$ 's in the left half-plane would imply the analyticity of $R . R_{2}$ and $R_{3}$ are analytic there by [21] and [3], respectively. The required analyticity of $R_{1}=R_{n-1, n+1}$ occurs if the denominator $Q_{n-1, n+1}$ has no zeros in the left half-plane. But $Q_{n-1, n+1}(z)=P_{n+1, n-1}(-z)$, and so it is sufficient to show that all the zeros of $P_{n+1, n-1}$ are in the left half-plane.

We proceed as in [3].

According to Wimp [23] all the zeros of the Bessel polynomial

$$
P_{n}^{(a)}(z)=\sum_{k=0}^{n}\left(\begin{array}{l}
n \\
k
\end{array}\right)(n+a)_{k} z^{n-k}, \quad a \geqslant 0, n \geqslant 1,
$$

where $(n+a)_{0}=1,(n+a)_{k}=(n+a)(n+a+1) \cdots(n+a+k-1)$ for $k>$ 1 , lie in the left half-plane. Because

$$
P_{n+1, n-1}(z)=\sum_{k=0}^{n-1} \frac{(2 n-k) !(n-1) !}{(2 n) ! k !(n-1-k) !} z^{k}=\frac{(n+1) !}{(2 n) !} P_{n-1}^{(3)}(z),
$$

it follows that $R_{1}$, and consequently $R$, are analytic in the left half-plane.

Let $R=P / Q$. When $z=$ it the definitions of $P_{n, m}$ and $Q_{n, m}$ and the identity $d_{1}=d_{3}$ imply

$$
\begin{aligned}
\left|Q\left(i t ; d_{1}, d_{2}, d_{3}\right)\right|^{2}-\left|P\left(i t ; d_{1}, d_{2}, d_{3}\right)\right|^{2} \\
=\left|Q_{n-1, n+1}\left(\mathrm{id}_{1} t\right) Q_{n, n}\left(\mathrm{id}_{2} t\right) Q_{n+1, n-1}\left(\mathrm{id}_{3} t\right)\right|^{2} \\
\quad-\left|P_{n-1, n+1}\left(\mathrm{id}_{1} t\right) P_{n, n}\left(\mathrm{id}_{2} t\right) P_{n+1, n-1}\left(\mathrm{id}_{3} t\right)\right|^{2} \equiv 0
\end{aligned}
$$


Hence the modulus of the rational function $R(z)=P\left(z ; d_{1}, d_{2}, d_{3}\right) / Q\left(z ; d_{1}, d_{2}, d_{3}\right)$ is one when $z$ is on the imaginary axis, which completes the proof of the lemma.

(d) $R_{1}=R_{n, n}, R_{2}=R_{n+1, n-1}, R_{3}=R_{n+2, n-2}, n \geqslant 2$.

Once again (3.1) shows the existence of a CEA of order $2 n+2$, while by (2.2), (2.3) we find the coefficients

$$
d_{1}=d_{1}^{*} /\left(1+d_{1}^{*}+d_{2}^{*}\right), \quad d_{2}=d_{2}^{*} /\left(1+d_{1}^{*}+d_{2}^{*}\right), \quad d_{3}=1 /\left(1+d_{1}^{*}+d_{2}^{*}\right),
$$

where

$$
\begin{aligned}
& d_{1}^{*}=\left(\frac{(n+1)(n+2)}{n(n-1)}\left(2\left(\frac{n-1}{2(n+2)}\right)^{1 /(2 n+2)}-1\right)\right)^{1 /(2 n+1)}, \\
& d_{2}^{*}=(2(n+2) /(n-1))^{1 /(2 n+2)} .
\end{aligned}
$$

This CEA is $A_{0}$-acceptable.

Let us now turn our attention to exponential fitting. By Theorem 5 and [8], if $R_{1}=R_{n, m}$ and $R_{2}=R_{n+1, m-1}$, there is a CEA of order $n+m$ that can be exponentially fitted to an arbitrary negative argument (because $\left\{R_{n, m}, R_{n+1, m-1}\right\}$ is a dominant pair). If $n=m$ then the CEA is $A$-acceptable.

Hence we can obtain exponentially fitted $L$-acceptable CEA of order $2 n$ with denominators of degree $n$ and $n+1$. For computational reasons we are interested in denominators of degrees as small as possible. The following example shows that if we settle for $A$-acceptability then, under some restrictions, we can obtain exponentially fitted CEA of order $2 n$, where the denominator of each rational function is of degree $n$.

(e) Let

$$
\begin{aligned}
& R_{1}(x ; \alpha)=\frac{(1-\alpha) P_{n, n-1}(x)+\alpha P_{n, n}(x)}{(1-\alpha) Q_{n, n-1}(x)+\alpha Q_{n, n}(x)}, \\
& R_{2}(x ; \alpha)=\frac{(1-\alpha) P_{n-1, n}(x)+\alpha P_{n, n}(x)}{(1-\alpha) Q_{n-1, n}(x)+\alpha Q_{n, n}(x)} .
\end{aligned}
$$

If $\alpha \in[0,1]$ then, by [4], $R_{1}$ is $A$-acceptable.

Furthermore, both $R_{k}$ 's are of order $2 n-1$ for any $\alpha$. We will form a CEA of order $2 n$ from $R_{1}$ and $R_{2}$. Its properties are given in Theorem 9 , which depends on the following lemma.

LEMMA 8. For every $\alpha$ in $[0,1]$ the function $Q(x ; \alpha)=(1-\alpha) Q_{n-1, n}(x)+$ $\alpha Q_{n, n}(x)$ has no zeros for $\operatorname{Re} x \leqslant 0$.

Proof. First we use a technique from the proof of Lemma 7 to show that $Q_{n-1, n}$ has no zeros in the left half-plane. The function value $Q_{n-1, n}(z)$ is equal to $P_{n, n-1}(-z)$, and the equation

$$
P_{n, n-1}(z)=\frac{n !}{(2 n-1) !} P_{n-1}^{(2)}(z)
$$

holds, where $P_{n-1}^{(2)}$ is a Bessel polynomial. Therefore by [23] all the zeros of $Q_{n-1, n}$ are in the right half-plane. 
We continue as in [5]; by deducing a contradiction from the assumption that $\alpha^{*}$ in $(0,1)$ and $z^{*}, \operatorname{Re} z^{*} \leqslant 0$, exist so that $Q\left(z^{*} ; d^{*}\right)=0$. Because $Q(z ; 1)=Q_{n, n}(z)$ $\neq 0$ and $Q(z ; 0)=Q_{n-1, n}(z) \neq 0$ for every $z$ in the left half-plane, the assumption and the root locus property [12] imply the existence of real numbers $\tilde{t}$ and $\tilde{\alpha}$, where $\tilde{\alpha}$ is in $(0,1)$, such that $Q(i \tilde{t} ; \tilde{\alpha})=0$. Hence the ratio $Q_{n, n}(i \tilde{t}) / Q_{n-1, n}(i \tilde{t})$ is real; in other words the equation $\operatorname{Im} Q_{n, n}(i \tilde{t}) / Q_{n-1, n}(i \tilde{t})=0$ holds, which is equivalent to the condition

$$
Q_{n, n}(i \tilde{t}) \overline{Q_{n-1, n}(i \tilde{t})}-Q_{n, n}\left(i t \overline{Q_{n-1, n}(i \tilde{t})}=0 .\right.
$$

Because $Q_{n-1, n}(-i \tilde{t})$ and $Q_{n, n}(-i \tilde{t})$ are equal to $P_{n, n-1}(i \tilde{t})$ and $P_{n, n}(i \tilde{t})$, respectively, and because the equations

$$
\begin{aligned}
& P_{n, n-1}(x)=P_{n, n}(x)-\frac{1}{2(2 n-1)} x P_{n-1, n-1}(x), \\
& Q_{n-1, n}(x)=Q_{n, n}(x)+\frac{1}{2(2 n-1)} x Q_{n-1, n-1}(x),
\end{aligned}
$$

are given in [5], it follows that the identity

$$
Q_{n, n}(i \tilde{t}) P_{n-1, n-1}(i \tilde{t})+P_{n, n}(i \tilde{t}) Q_{n-1, n-1}(i \tilde{t})=0
$$

is obtained, which is the same as the equation

$$
\operatorname{Re} Q_{n, n}(i \tilde{t}) P_{n-1, n-1}(i \tilde{t})=0 .
$$

The polynomial $\operatorname{Re} Q_{n, n}(i \tilde{t}) P_{n-1, n-1}(i \tilde{t})$ is even and, according to [5], the evenpowered terms of $Q_{n, n}(z) P_{n-1, n-1}(z)$ are

$$
E_{n, n-1}(z)=\frac{2 n !(n-1) !}{(2 n-2) !(2 n) !} \sum_{j=0}^{n-1} \frac{(2 n-2 j-2) !(2 n-j-1) !}{j !((n-j-1) !)^{2}}\left(-z^{2}\right)^{j}
$$

Hence $\operatorname{Re} Q_{n, n}(i \tilde{t}) P_{n-1, n-1}(i \tilde{t})=E_{n, n-1}(i \tilde{t})>0$. This contradiction completes the proof.

THEOREM 9. Let $R_{1}$ and $R_{2}$ be as defined above and let $R=R(x ; \alpha)$ be the corresponding $C E A$ with $d_{1}=d_{2}=\frac{1}{2}$. Then

(i) $R$ is $A$-acceptable for every $\alpha$ in $[0,1]$.

(ii) For every $\alpha, R(x ; \alpha)-\exp (x)=O\left(x^{2 n+1}\right)$.

(iii) $x_{0}<0$ exists such that, for every $x^{*} \leqslant x_{0}$, there is an $\alpha=\alpha\left(x^{*}\right)$ in $[0,1]$ so that $R\left(x^{*} ; \alpha\right)=\exp \left(x^{*}\right)$.

Proof. (i) By Lemma 8 and the $A$-acceptability of $R_{1}, R$ is analytic in the left half-plane. Furthermore, for every $\alpha$ and real $t,|R(i t ; \alpha)| \equiv 1$. The acceptability follows.

(ii) The order of $R$ is proved by combining Theorem 3 and Lemma 6, using the identity $Q_{n, m}(0)=1$.

(iii) By examining coefficients we find that, for $x \ll 0, R(x ; 0)<0<\exp (x)$. Moreover, because $R(x ; 1)=R_{n, n}^{2}\left(\frac{1}{2} x\right)>0$, and because the decay of rational functions is necessarily slower than that of $\exp (x)$, we deduce $R(x ; 1)>\exp (x)$ when $x \ll 0$.

Therefore $x_{0}<0$ exists such that, for every $x \leqslant x_{0}$,

$$
R(x ; 0)<\exp (x)<R(x ; 1) \text {. }
$$


Since $R$ is continuous in $\alpha$ for $\alpha \in[0,1]$, it follows that, for every $x^{*} \leqslant x_{0}$, $\alpha=\alpha\left(x^{*}\right)$ exists such that $R\left(x^{*}, \alpha\right)=\exp \left(x^{*}\right)$, which is the required result.

Table I gives the greatest $x_{0}$ that is allowed by Theorem 9 for $1 \leqslant n \leqslant 10$.

TABLE I. The $x_{0}$ 's for $n=1, \ldots, 10$.

\begin{tabular}{|r|r|}
\hline$n$ & \multicolumn{1}{|c|}{$x_{0}$} \\
\hline 1 & -4.7987 \\
2 & -5.5882 \\
3 & -10.0729 \\
4 & -10.9024 \\
5 & -15.3653 \\
6 & -16.2086 \\
7 & -20.6624 \\
8 & -21.5127 \\
9 & -25.9615 \\
10 & -26.8159 \\
\hline
\end{tabular}

Remark. The principal error term (i.e., the coefficient of $x^{2 n+1}$ in the error expansion) of $R(x ; \alpha)$ has the value

$$
E_{n}(\alpha)=\frac{(-1)^{n-1}(n !)^{2}}{2^{2 n}((2 n) !)^{2}(2 n-1)}\left(\frac{4 n}{2 n+1}-2 \alpha+\alpha^{2}\right) .
$$

Because $\alpha^{2}-2 \alpha+4 n /(2 n+1) \neq 0$ for every $n \geqslant 1$ and real $\alpha$, the order $2 n$ cannot be increased. Furthermore, $\left|E_{n}(\alpha)\right|$ attains its minimum value when $\alpha=1$, which corresponds to two equal steps by the diagonal Padé approximation $R_{n, n}$. Therefore, as far as order and local error (but not exponential fitting) are concerned, one cannot do better than the diagonal Padé approximation.

Remark. Higher orders of the CEA can be attained by allowing complex $d_{k}$ 's, complex $R_{k}$ 's or $R_{k}$ 's such that

$$
R_{k}(x)-\exp (x)=a_{k} x+O\left(x^{2}\right), \quad a_{k} \neq 0 .
$$

Three examples are given:

(i) If $R_{1}=R_{2}=R_{1,1}, d_{1}=\frac{1}{2}+\sqrt{3} i / 6, d_{2}=\frac{1}{2}-\sqrt{3} i / 6$, then $R\left(x ; d_{1}, d_{2}\right)=$ $R_{2,2}(x)$, of order 4 .

(ii) If $\alpha=1 \pm \sqrt{(2 n-1) /(2 n+1) i}$, then the CEA of Theorem 8 is of order $2 n+1$ (as can be easily verified by an examination of the principal error term).

(iii) If $s=\sqrt[3]{4(\sqrt{5}+1)}-\sqrt[3]{4(\sqrt{5}-1)}$

$$
\begin{aligned}
q_{1}(x) & =1-\frac{2}{4+s} x, \\
q_{2}(x) & =1-\frac{16-2 s}{16-4 s+s^{2}-6 \sqrt[3]{2}} x+\frac{4}{16-4 s+s^{2}-6 \sqrt[3]{2}} x^{2}, \\
R_{1}(x) & =q_{1}(-x) / q_{1}(x), \quad R_{2}(x)=q_{2}(-x) / q_{2}(x), \text { then } \\
R\left(x ; \frac{1}{2}, \frac{1}{2}\right) & =R_{3,3}(x), \text { of order } 6 .
\end{aligned}
$$

However, these approximations are of little or no computational value. 
4. Nørsett Approximations. Rational approximations to $\exp (x)$ with only real poles [17], [18] are of great importance in the numerical solution of large stiff systems with sparse Jacobian matrices (such systems are obtained, for example, when one solves parabolic partial differential equations by the method of lines). They are variously called multiple Padé approximations [17], $N$-approximations [18], and restricted Padé approximations [22]. In the following we prefer the name $N$-approximations.

According to Nørsett and Wolfbrandt [18], one cannot exceed order $n+1$ if an approximation has $n$ poles, which are all real and the degree of the numerator does not exceed $n$. The best such approximation, as far as local principal error is concerned, is attained by an approximation that has just one real pole, of multiplicity $n$. Therefore, in the sequel we restrict our attention to this type of approximation.

Let $L_{n}^{(d)}$ denote the generalized Laguerre polynomial [19],

$$
L_{n}^{(d)}(x)=\frac{(1+d)_{n}}{n !} \sum_{k=0}^{n}(-1)^{k}\left(\begin{array}{l}
n \\
k
\end{array}\right) \frac{1}{(1+d)_{k}} x^{k},
$$

where $(1+d)_{0}=1,(1+d)_{k}=(1+d)(2+d) \cdots(k+d)$. The usual Laguerre polynomial of degree $n$ is $L_{n}=L_{n}^{(0)}$.

Let $S_{n, k}(x)=p_{n, k}(x) /\left(1-a_{k} x\right)^{n}$, where

$$
p_{n, k}(x)=\sum_{i=0}^{n}\left(\sum_{j=0}^{i} \frac{(-1)^{i}}{(i-j) !}\left(\begin{array}{l}
n \\
j
\end{array}\right) a_{k}^{j}\right) x^{i},
$$

and where $a_{k}$ is the reciprocal of the $k$ th zero of $L_{n}^{(1)}$ (and so, according to [19], $a_{k}$ is real and positive). Nørsett [17] proves that $S_{n, k}(x)-\exp (x)=c_{n, k} x^{n+2}+O\left(x^{n+3}\right)$, where

$$
c_{n, k}=(-1)^{n+1} \frac{1}{n+2} a_{k}^{n+1} L_{n}\left(1 / a_{k}\right) .
$$

We consider in this section some CEA's that are formed from these $N$-approximations.

THEOREM 10. The pair $\left\{S_{n, i_{1}}, S_{n, i_{2}}\right\}$ is dominant if and only if $i_{1}+i_{2}$ is odd.

Proof. According to Theorem 4.3 of [17],

$$
S_{n, k}(x)-e^{x}=L_{n}\left(1 / a_{k}\right) e^{x} \int_{0}^{x}\left(t /\left(t-1 / a_{k}\right)\right)^{n+1} e^{-t} d t .
$$

Because $a_{k}>0$, the integral on the right is positive for $x<0$. Hence it is sufficient to prove that the signs of the numbers $\left\{L_{n}\left(1 / a_{k}\right): k=1,2, \ldots, n\right\}$ alternate. According to [19],

$$
L_{n}(x)=L_{n}^{(1)}(x)-L_{n-1}^{(1)}(x) .
$$

Therefore the proof follows by the interlacing property of the zeros of $\left\{L_{n}^{(1)}\right\}_{n=0}^{\infty}$.

(f) Let $R_{1}=S_{n, i_{1}}$ and $R_{2}=S_{n, i_{2}}$. Because sgn $c_{n, i_{1}} c_{n, i_{2}}=(-1)^{i_{1}+i_{2}}$, it follows that, if $i_{1}+i_{2}$ is odd, then $d_{1}$ and $d_{2}$ in $(0,1)$ exist, such that the CEA is of order $n+2$. In fact, according to Theorem 2 ,

$$
d_{1}=d^{*} /\left(1+d^{*}\right) ; \quad d_{2}=1 /\left(1+d^{*}\right)
$$


where

$$
d^{*}=\left(-\left(a_{i_{2}}^{n+1} L_{n}\left(1 / a_{i_{2}}\right)\right) /\left(a_{i_{1}}^{n+1} L_{n}\left(1 / a_{i_{1}}\right)\right)\right)^{1 /(n+2)} .
$$

According to [22] very few $N$-approximations of the type $S_{n, k}$ are $A$-acceptable. However, owing to the special structure of the sparse Jacobian matrices, which are encountered in the numerical solution of parabolic equations by the method of lines, $A_{0}$-acceptability is frequently a suitable stability requirement.

The $A_{0}$-acceptable approximations $S_{n, k}$ are fully characterized in [17]. The following lemma gives a stability result which, although less general, is sufficient for many practical cases of CEA's of two approximations $S_{n, k}$.

LEMMA 11. Let $R_{k}=S_{n, i_{k}}, i_{1}+i_{2}$ odd and $b_{k}=L_{n}\left(1 / a_{i_{k}}\right), k=1,2$, where $a_{i_{k}}$ is the $i_{k}$ th zero of $L_{n}^{(1)}(1 / x)$, and let $R$ be any $C E A$. Then

(i) $\left|b_{1} b_{2}\right| \leqslant 1$ is necessary for the $A_{0}$-acceptability of $R$.

(ii) If $\left|b_{1}\right|,\left|b_{2}\right| \leqslant 1$, then $R$ is $A_{0}$-acceptable.

Proof. (i) According to (4.1) for $x \ll 0$

$$
S_{n, i_{k}} \approx(-1)^{n} \sum_{j=0}^{n} \frac{(-1)^{j}}{(n-j) !}\left(\begin{array}{l}
n \\
j
\end{array}\right) a_{i_{k}}^{j} / a_{i_{k}}^{n}=b_{k} .
$$

Hence for $d_{1}, d_{2} \in(0,1)$

$$
\lim _{x \rightarrow-\infty}\left|R\left(x ; d_{1}, d_{2}\right)\right|=\left|b_{1} b_{2}\right|
$$

which shows that $R$ is $A_{0}$-acceptable only if $\left|b_{1} b_{2}\right| \leqslant 1$.

(ii) If $\left|b_{1}\right|,\left|b_{2}\right| \leqslant 1$, then, according to [17], both $S_{n, i_{1}}$ and $S_{n, i_{2}}$ are $A_{0}$-acceptable. The $A_{0}$-acceptability of $R$ follows from Lemma 1 and Theorem 3 .

Table II gives the acceptability properties of some composite $N$-approximations of type ( $f$ ) and, for the sake of comparison, the acceptability properties of some $N$-approximations $S_{n, k}$. According to [17], the $A_{0}$-acceptable $N$-approximations are also $A(\alpha)$-acceptable, where $R$ is $A(\alpha)$-acceptable if $|R(z)|<1$ for every $z$ which belongs to the wedge-shaped domain $\{z:|\arg (-z)|<\alpha\}$ of the complex left half-plane [11]. It is customary to present $\alpha$ in degrees, rather than in radians-thus $A(90)$-acceptability corresponds to $A$-acceptability. As Nørsett points out in [17], for $n \leqslant 9$ the $A_{0}$-acceptable $N$-approximations are $A(\alpha)$-acceptable with $\alpha>89$. As is evident from Table II, the composition preserves this property.

(g) $R_{k}=S_{n, i_{k}}, k=1,2, i_{1}+i_{2}$ odd, with exponential fitting.

As a consequence of Theorem 10 , for every $x_{0}<0, d$ exists in $(0,1)$ such that $R\left(x_{0} ; d, 1-d\right)=\exp \left(x_{0}\right)$. If $x_{0}<0$ then the order is $n+1$, while, in the limit as $x_{0}$ tends to zero, one gets the CEA of type (f).

(h) $R_{k}=S_{n, i_{k}}, k=1,2,3$.

Table II shows the relative scarcity of acceptable $N$-approximations and their composite counterparts for small values of $n$. The least value of $n$ for which there is an $A_{0}$-acceptable CEA of three $N$-approximations, whose order is $n+3$, is $n=6$, and the coefficients of the CEA are $i_{1}=4, i_{2}=5, i_{3}=6, d_{1}=0.59375, d_{2}=$ $0.34375, d_{3}=0.06250$. 
TABLe II

All the acceptable $N$-approximations $S_{n, i}$ and $C E A\left\{S_{n, i_{1}}, S_{n, i_{2}}\right\}$ for $2 \leqslant n<7$

\begin{tabular}{c|c|c|c|c|c|c}
\hline$n$ & $i_{1}$ or $i$ & $i_{2}$ & $d_{1}$ & $d_{2}$ & Acceptability & Order \\
\hline 2 & 2 & - & - & - & $A$-acc. & 3 \\
& 1 & 2 & 0.341081377 & 0.658918623 & $A$-acc. & 4 \\
\hline 3 & 3 & - & - & - & $A$-acc. & 4 \\
& 2 & 3 & 0.301016914 & 0.698983084 & $A$-acc. & 5 \\
\hline 4 & 4 & - & - & - & $A$-acc. & 5 \\
& 3 & 4 & 0.281617072 & 0.718382928 & $A$ (89)-acc. & 6 \\
\hline 5 & 4 & - & - & - & $A$-acc. & 6 \\
& 5 & - & - & - & $A(89)$-acc. & 6 \\
& 4 & 5 & 0.270247623 & 0.729752377 & $A(89)$-acc. & 7 \\
\hline 6 & 5 & - & - & - & $A(89)$-acc. & 7 \\
& 6 & - & - & - & $A(89)$-acc. & 7 \\
& 5 & 6 & 0.262827198 & 0.737172802 & $A(89)$-acc. & 8 \\
\hline 7 & 6 & - & - & - & $A(89)$-acc. & 8 \\
& 7 & - & - & - & $A(89)$-acc. & 8 \\
& 5 & 6 & 0.354081725 & 0.645918275 & $A$-acc. & 9 \\
& 6 & 7 & 0.257628647 & 0.742371353 & $A(89)$-acc. & 9 \\
\hline
\end{tabular}

An alternative approach to the $N$-approximations [17] is to let the polynomial $p$ in the numerator be of degree $n-1$ and to require the order of the approximation to be only $n$. This approach is of relevance to semiexplicit Runge-Kutta methods [16], [1]. As is conjectured by Nørsett [16] no semiexplicit Runge-Kutta method of $n$ stages can attain order $n+1$ for even $n, n \geqslant 4$. The exponential approximations of these methods are $N$-approximations. Among the $N$-approximations of order $n$ the approximations with $\operatorname{deg} p=n-1$ have the best asymptotic acceptability properties.

It is proven in [17] that the approximation

$$
T_{n, k}(x)=p_{n, k}(x) /\left(1-a_{k} x\right)^{n}
$$

is of order $n$, where $P_{n, k}$ is the polynomial (4.1) and where $a_{k}$ is the reciprocal of the $k$ th zero of $L_{n}$. The choice of $a_{k}$ implies $\operatorname{deg} p=n-1$ and $T_{n, k}(x)-\exp (x)=$ $c_{n, k} x^{n+1}+O\left(x^{n+2}\right)$, where

$$
c_{n, k}=\frac{(-1)^{n+1}}{n+1} a_{k}^{n} L_{n}^{(1)}\left(1 / a_{k}\right)
$$

THEOREM 12. The pair $\left\{T_{n, i_{1}}, T_{n, i_{2}}\right\}$ is dominant if and only if $i_{1}+i_{2}$ is odd.

Proof. According to Theorem 4.3 in [17]

$$
T_{n, k}(x)-e^{x}=L_{n}^{(1)}\left(1 / a_{k}\right) / a_{k} e^{x} \int_{0}^{x} t^{n} /\left(t-1 / a_{k}\right)^{n+1} e^{-t} d t .
$$

The proof proceeds along the same lines as the proof of Theorem 10.

Hence, by Theorems 3 and 5 it is possible to form CEA of the $N$-approximations $T_{n, k}$ in order to exponentially fit or increase the order, while preserving the acceptability properties. 
Acknowledgement. The author would like to express his gratitude to Professor $\mathbf{M}$. J. D. Powell of Cambridge University, who has read the manuscript of this paper and very kindly suggested many improvements.

\section{King's College}

University of Cambridge

Cambridge CB2 1ST, England

1. R. AleXANDeR, "Diagonally implicit Runge-Kutta methods for stiff O.D.E.'s," SIAM J. Numer. Anal., v. 14, 1977, pp. 1006-1021.

2. J. Donelson \& E. HANSEN, "Cyclic composite multistep predictor-corrector methods," SIAM J. Numer. Anal., v. 8, 1971, pp. 137-157.

3. B. L. EhLE, " $A$-stable methods and Pade approximations to the exponential," SIAM J. Math. Anal., v. 4, 1973, pp. 671-680.

4. B. L. EHLE, Some Results on Exponential Approximation and Stiff Equations, Report 77, Dept. of Math., Univ. of Victoria, Canada, 1974.

5. B. L. EHLE \& Z. PICRL, "Two-parameter, arbitrary order, exponential approximations for stiff equations," Math. Comp., v. 29, 1975, pp. 501-511.

6. C. W. Gear, Numerical Initial Value Problems in Ordinary Differential Equations, Prentice-Hall, Englewood Cliffs, N. J., 1971.

7. H. GreEnSPAN, W. HAFNer \& M. Ribaric, "On varying stepsizes in numerical integration of first order differential equations," Numer. Math., v. 7, 1965, pp. 286-291.

8. A. ISERLES, “ $A$-stability and dominating pairs," Math. Comp., v. 32, 1978, pp. 19-33.

9. A. ISERLES, "Nonexponential fitting techniques for numerical solution of stiff equations," Utilitas Math., v. 17, 1980, pp. 276-302.

10. A. IsERLes, "On the generalized Pade approximations to the exponential function," SIAM J. Numer. Anal., v. 16, 1979, pp. 631-636.

11. R. JeLTSCH, "Stiff stabillity and its relation to $A_{0}$ - and $A(0)$-stability," SIAM J. Numer. Anal., v. 13,1976, pp. 8-17.

12. A. M. KRALL, "The root locus method: $A$ survey," SIAM Rev., v. 12, 1970, pp. 64-72.

13. B. LINDBERG, "Characterization of optimal stepsize sequences for methods for stiff differential equations," SIAM J. Numer. Anal., v. 14, 1977, pp. 859-887.

14. W. Liniger \& R. A. WILLOUGHBY, "Efficient integration methods for stiff systems of ordinary differential equations," SIAM J. Numer. Anal., v. 7, 1970, pp. 47-66.

15. D. Morrison, "Optimal mesh size in the numerical integration of an ordinary differential equation," J. Assoc. Comput. Mach., v. 9, 1962, pp. 98-103.

16. S. P. NøRSETT, Semi-Explicit Runge-Kutta Methods, Report 6, Dept. of Math., Univ. of Trondheim, Norway, 1974.

17. S. P. NøRSETT, Multiple Padé Approximations to the Exponential Function, Report 4, Dept. of Math., Univ. of Trondheim, Norway, 1974; (appeared in an abridged form as "Restricted Padé approximations to the exponential functions," SIAM J. Numer. Anal., v. 15, 1978, pp. 1008-1029).

18. S. P. NøRSETT \& A. WolFBRANDT, "Attainable order of rational approximations to the exponential function with only real poles," BIT, v. 17, 1977, pp. 200-208.

19. E. D. RaInville, Special Functions, Macmillan, New York, 1967.

20. H. J. STETTER, Analysis of Discretization Methods for Ordinary Differential Equations, SpringerVerlag, Berlin, 1973.

21. R. S. VARGA, "On higher order stable implicit methods for solving parabolic partial differential equations," J. Math. Phys., 1961, pp. 220-231.

22. G. WANNER, E. HAIRER \& S. P. NøRSETt, “Order stars and stability theorems," BIT, v. 18, 1978, pp. 475-489.

23. J. WMM, "On zeros of a confluent hypergeometric function," Proc. Amer. Math. Soc., v. 16, 1965, pp. 281-283. 